El mercado laboral mexicano ante las propuestas del ODS 8: generar trabajo decente y crecimiento económico

The Mexican labor market in view of the SDG 8 proposals: generate decent work and economic growth

- Óscar Peláez Herreros

El Colegio de la Frontera Norte, México

Fecha de recepción: 16 de marzo de 2021

Fecha de aprobación: 7 de mayo de 2021

DOI: http://dx.doi.org/10.15304/ricd.3.14.7582

\title{
NOTAS BIOGRÁFICAS
}

Óscar Peláez Herreros es licenciado y doctor en Economía por la Universidad de Cantabria, España. Investigador titular del Departamento de Estudios Económicos de El Colegio de la Frontera Norte, sede Tijuana, México. Es miembro del Sistema Nacional de Investigadores de México, nivel 2.

Contacto: opelaez@colef.mx

\section{Resumen}

El objetivo de este artículo es identificar los retos y dificultades a los que se enfrenta México para atender al octavo Objetivo de Desarrollo Sostenible (ODS), que busca promover el trabajo decente y el crecimiento económico. Para ello, se describen y explican las principales características del mercado laboral mexicano (bajas tasas de desempleo, altos porcentajes de informalidad, bajos salarios e importantes diferencias interregionales) y se analizan las metas específicas del ODS 8. La información obtenida de diversas fuentes (Banco Mundial, Organización Internacional del Trabajo, Organización para la Cooperación y el Desarrollo Económicos, Inegi, Coneval y algunas Secretarías del Gobierno de México) permite conocer el estado de avance y los rezagos que quedan por atender en cada meta. Con ello, se muestra que, desde la década de los ochenta, la economía mexicana ha crecido a un ritmo muy lento, en concreto, a una tasa del 0,5\% promedio anual. Ese escaso crecimiento se debe en su mayor parte al incremento del tamaño relativo de la fuerza de trabajo y en menor medida al aumento de la productividad. De hecho, en 2019, el producto por trabajador era un 1,5\% inferior al del año 2000. En las últimas décadas, el mercado de trabajo formal no fue capaz de generar suficientes empleos, que entonces surgieron en el sector informal ya que gran parte de la población necesita disponer de algún ingreso para subsistir. Los problemas de estancamiento de la productividad y lento crecimiento de la economía se ven acompañados por: desincentivos para la formalización de actividades, desempleo juvenil, limitada reducción del trabajo infantil, altas tasas de lesiones ocupacionales mortales, y escasa inclusión financiera especialmente en la región sur del país. Por lo mencionado, se concluye que tomar medidas para elevar la productividad laboral es clave para empezar a corregir estos problemas y generar empleos decentes. 


\section{Abstract}

The objective of this paper is to identify the challenges and difficulties that Mexico faces in meeting the eighth Sustainable Development Goal (SDG), which seeks to promote decent work and economic growth. To do this, we describe and explain the main characteristics of the Mexican labor market (low unemployment rates, high percentages of informality, low wages and significant differences between regions) and analyze the specific targets of SDG 8. The information from various sources (World Bank, International Labor Organization, Organization for Economic Cooperation and Development, Inegi, Coneval and some Secretariats of the Government of Mexico) allows us to know the progress status and the lags that remain to be addressed in each target. Thus, we find that, since the 1980s, the Mexican economy has grown at a very slow pace, specifically, at an average annual rate of $0.5 \%$. This low growth is due mainly to the increase in the relative size of the labor force and to a lesser extent to the increase in productivity. In fact, in 2019, the product per worker was $1.5 \%$ lower than in 2000. In recent decades, the formal labor market was not able to generate enough jobs, which then emerged in the informal sector since a large part of the population needs to have some income to survive. The problems of productivity stagnation and slow economic growth are accompanied by: disincentives to formalize activities, youth unemployment, limited reduction in child labor, high rates of fatal occupational injuries, and low financial inclusion, especially in the southern region of the country. Therefore, we conclude that taking measures to increase labor productivity is very important to correct these problems and generate decent jobs.

\section{Palabras clave}

Objetivos de Desarrollo Sostenible, desempleo, informalidad laboral, trabajo decente, productividad, crecimiento económico, diferencias interregionales.

\section{Keywords}

Sustainable Development Goals, unemployment, labor informality, decent work, productivity, economic growth, interregional differences.

\section{Sumario}

1. Introducción

2. Aspectos fundamentales del mercado laboral mexicano

3. Las metas del objetivo de trabajo decente y crecimiento económico de los ODS

4. Reflexiones finales

\section{Summary}

1. Introduction

2. Fundamental issues of the Mexican labor market

3. The SDG tarjets for decent work and economic growth

4. Final thoughts 


\section{INTRODUCCIÓN}

En palabras de Naciones Unidas (2018, p. 5), la Agenda 2030 para el Desarrollo Sostenible, aprobada en septiembre de 2015, "presenta una oportunidad histórica para América Latina y el Caribe, ya que incluye temas altamente prioritarios para la región, como la erradicación de la pobreza extrema, la reducción de la desigualdad en todas sus dimensiones, un crecimiento económico inclusivo con trabajo decente para todos, ciudades sostenibles y cambio climático, entre otros." Concretamente, la Agenda se compone de 17 Objetivos de Desarrollo Sostenible (ODS) que dan cierta continuidad a los Objetivos de Desarrollo de Milenio (ODM).

Sin desmerecer al resto de objetivos, el presente artículo se centra en el análisis del octavo de ellos, que trata sobre "trabajo decente y crecimiento económico", prestando atención a la coherencia y viabilidad de sus metas para el caso de México. Cabe considerar que los ODS son una propuesta de las Naciones Unidas a nivel mundial, y que México no es uno de los países peor posicionados para atender los objetivos marcados. No obstante, como se expone a continuación, algunas metas resultan prácticamente inalcanzables y carecen de realismo en su planteamiento.

Para mostrar esta situación, en primer lugar, se describen las principales características del mercado laboral mexicano. Como se podrá comprobar, estas distan bastante de las de otros países. Las bajas tasas de desempleo, el alto porcentaje de informalidad o las fuertes diferencias entre regiones son algunos de los aspectos que caracterizan la situación y condicionan la posible evolución de los problemas relacionados con el mercado de trabajo. En el tercer apartado se analizan las metas del objetivo 8 de los ODS, que busca promover el trabajo decente y el crecimiento económico. Se verá que, precisamente, la ausencia de crecimiento económico que se prolonga desde la década de los ochenta está detrás de los problemas que acumula el mercado de trabajo formal, incapaz de crear suficientes empleos de calidad. El artículo concluye con un apartado de reflexiones que retoma los puntos más relevantes que vinculan los problemas específicos del mercado laboral mexicano con las propuestas de los ODS.

\section{ASPECTOS FUNDAMENTALES DEL MERCADO LABORAL MEXICANO}

En 2019, México era uno de los países de la Organización para la Cooperación y el Desarrollo Económicos (OCDE) con menor tasa de desempleo (o desocupación) (TD). Apenas el 3,49\% de la población económicamente activa (PEA) clasificaba en esa situación (OCDE, 2021). ${ }^{1}$ Japón $(2,35 \%)$, Alemania $(3,15 \%)$ o los Países Bajos $(3,40 \%)$ mostraban cifras ligeramente menores, mientras que, en la situación opuesta, destacaban Colombia (10,55\%), Turquía (13,70\%), España $(14,12 \%)$ y Grecia $(17,33 \%)$ evidenciando amplios desequilibrios en sus mercados laborales. Esta característica no es coyuntural. La TD de México presenta sistemáticamente valores bajos. De 1987 a 2020, se mantuvo entre el 6,27\% observado en 1995 y el $2,51 \%$ de 1999 . Ni siquiera en la crisis de 2008-09 se elevó más allá del $5,48 \%$.

Esta aparente eficacia estructural para mantener ocupada a la mano de obra contrasta con los altos niveles de pobreza que padece la población. Según cifras oficiales, el 41,91\% de los mexicanos son pobres (Coneval, 2019). El $16,81 \%$ ni siquiera disponen de ingresos suficientes para cubrir su alimentación básica. Con ello, surge la situación paradójica de que, habiendo empleo y aun aplicando políticas específicas para mitigar la pobreza, esta persiste en niveles altos, afectando a casi la mitad de la población. La paradoja deja de serlo al considerar algunas particularidades del caso.

En primer lugar, hay que tener en cuenta que en México no existe seguro de desempleo. El artículo 123 de la Constitución hace referencia a un seguro "de cesación involuntaria del trabajo" que en la práctica cubre únicamente a los trabajadores mayores de sesenta años (Ochoa, 2005, p. 28). En la actualidad, sólo los trabajadores de la capital del país que pierden un empleo formal tienen la posibilidad de recibir un subsidio público, que consiste en 2.641 pesos (poco más de 100 euros) durante un máximo de 6 meses (Juárez, 2020).

Como explica Negrete (2011, p. 153), "al no existir un seguro de desempleo y tampoco los ahorros necesarios para estar desempleado mientras se consigue un trabajo, los desocupados se dedican a cualquier actividad que les represente un ingreso". El mercado laboral informal permite a estas personas realizar "alguna actividad económica durante al menos una hora" 
a la semana. Con ello, clasifican como "ocupados" según la definición estándar:

Población ocupada: Personas de 15 y más años de edad que en la semana de referencia realizaron alguna actividad económica durante al menos una hora. Incluye a los ocupados que tenían trabajo, pero no lo desempeñaron temporalmente por alguna razón, sin que por ello perdieran el vínculo laboral con este; así como a quienes ayudaron en alguna actividad económica sin recibir un sueldo o salario. (Inegi, 2020a).

Los trabajadores informales son los que se desempeñan "en una unidad económica no constituida en sociedad que opera a partir de los recursos de los hogares, y que no lleva un registro contable de su actividad" (Inegi, 2020a). Los vendedores ambulantes son el caso paradigmático. Pero también clasifican como informales los trabajadores que, por ejemplo, no están registrados ante la seguridad social aun aportando su fuerza de trabajo a una empresa formalmente constituida (Inegi, 2014, p. 9). Hay ocupaciones en el sector formal que operan bajo condiciones de informalidad. De esta manera, la ocupación se considera informal cuando, por el contexto en el que se realiza, el trabajador no puede "invocar a su favor el marco legal o institucional que corresponda a su inserción económica" (Inegi, 2014, p. 36).

En la práctica, los impedimentos para realizar actividades informales son mínimos. El Instituto Nacional de Estadística y Geografía (Inegi) calcula la tasa de informalidad laboral (TIL1), que mide el porcentaje de ocupados informales en la población ocupada (Inegi, 2020a). ${ }^{2}$ La cifra más reciente, la del tercer trimestre de 2020, indica que el $54,2 \%$ de los ocupados se encuentran en situación de informalidad, esto es, "carecen de la protección de la seguridad social básica por parte de su trabajo" o bien se emplean "en unidades económicas no registradas, situación que los coloca en una situación de vulnerabilidad para hacer valer las garantías laborales elementales" (Inegi, 2014, pp. 26-7). La TIL1 más alta desde 2005 corresponde al cuarto trimestre de 2009, cuando alcanzó el 60,0\%.

Ante una recesión económica, el mercado laboral se ajusta en términos de cantidad y calidad de empleos. Por las condiciones existentes en México, gran parte de la población no puede dejar de trabajar y de percibir algún ingreso. En consecuencia, una contracción de la producción no eleva sustancialmente la TD, sino que incide en otros indicadores, como la tasa de informalidad o el salario promedio. Sin embargo, la causa de los altos niveles de informalidad no está en los ciclos de la economía. La TIL1 no ha bajado del $50 \%$ ni siquiera durante las fases de crecimiento, evidenciando importantes problemas estructurales que sitúan a más de la mitad de los ocupados en condiciones de informalidad.

Ros (2019a, p. 17) explica que el lento crecimiento o "semi-estancamiento" de largo plazo característico de la economía mexicana desde la década de los ochenta se relaciona con el hecho de que el sector formal sea incapaz de generar puestos de trabajo suficientes para absorber "las grandes reservas de empleo informal con que cuenta la economía". Según datos del Banco Mundial (2021), el producto interno bruto (PIB) per cápita de México expresado en precios constantes creció a una tasa anual acumulativa de $3,77 \%$ entre 1960 y 1981 . Sin embargo, de 1981 a 2019 la tasa media se situó en 0,50\%. El crecimiento de los noventa $(1,74 \%)$ y de la década más reciente $(1,14 \%)$ apenas compensaron el decrecimiento de los ochenta $(-0,29 \%)$ y el estancamiento de la primera década del nuevo siglo $(0,02 \%)$. En este sentido, Okun (1962) ya advertía que ritmos de crecimiento del PIB relativamente bajos también generan desempleo.

Como se ha argumentado, en el caso de México, el lento crecimiento no ha impactado tanto en la TD como en la calidad de los empleos y en las condiciones laborales. La permanencia de la TIL1 por encima del 50\% se ha visto acompañada por otros aspectos que merecen atención, como el hecho de que seis de cada diez ocupados no cuenten con asistencia médica en instituciones de salud públicas o privadas. En cuanto a las remuneraciones, datos del Inegi (2020b) muestran que, en el cuarto trimestre de 2019 , el $51,43 \%$ de la población ocupada ingresaba menos de dos salarios mínimos, lo que representa alrededor de 290 euros al mes. El $6,03 \%$ ni siquiera recibía ingresos. Y únicamente el $3,61 \%$ de los ocupados ganaba más de cinco salarios mínimos (727 euros mensuales). Estas cifras no han mejorado en los últimos 15 años. Al contrario, en el cuarto trimestre de 2005, sólo el $35,45 \%$ de los ocupados recibía menos de dos salarios mínimos (218 euros de entonces), mientras que el 11,02\% obtenía más de cinco salarios mínimos.

Otra cuestión relevante es la referida a las diferencias interregionales. De acuerdo con lo mencionado, los estados con mayores cifras de pobreza y menores índices de desarrollo humano (IDH) son los que disponen de mercados laborales menos formalizados. Esto implica que padecen mayores tasas de informalidad, peores condiciones laborales y menores tasas de población asalariada y desocupación, no habiendo ninguna 
contradicción en esto último. Como menciona Negrete (2011, p. 146), “la tasa de desocupación recoge, en esencia, el comportamiento de los segmentos modernos de la fuerza laboral"; por ello, la TD es más baja donde el sector moderno es más reducido.

Tabla 1. Indicadores laborales para los tres estados con mayor y menor pobreza en 2018.

\begin{tabular}{l|lll|lll}
\hline & Chiapas & Guerrero & Oaxaca & Coahuila & $\begin{array}{l}\text { Baja California } \\
\text { Sur }\end{array}$ & Nuevo León \\
\hline \% de pobres & 76,4 & 66,5 & 66,4 & 22,5 & 18,1 & 14,5 \\
\hline IDH & 0,67 & 0,68 & 0,68 & 0,77 & 0,78 & 0,79 \\
\hline TIL1 & 71,8 & 79,3 & 80,5 & 34,8 & 38,7 & 36,8 \\
\hline $\begin{array}{l}\text { \% de ocupados con acceso a } \\
\text { instituciones de salud }\end{array}$ & 17,0 & 17,4 & 15,2 & 60,7 & 52,5 & 59,2 \\
\hline $\begin{array}{l}\text { Tasa de trabajo asalariado } \\
\text { \% de ocupados con ingreso } \\
\text { inferior a 2 salarios mínimos }\end{array}$ & 44,6 & 43,8 & 40,2 & 78,6 & 71,4 & 77,3 \\
\hline $\begin{array}{l}\text { \% de ocupados con ingreso } \\
\text { superior a 5 salarios } \\
\text { mínimos }\end{array}$ & 1,6 & 0,9 & 1,5 & 6,1 & 34,9 & 32,6 \\
\hline \begin{tabular}{l} 
TD \\
\hline
\end{tabular} & 2,5 & 1,4 & 2,0 & 4,3 & 9,5 & 9,0 \\
\hline
\end{tabular}

Fuente: Elaboración propia con datos de Coneval (2019) para la pobreza, PNUD (2015) para el IDH, e Inegi (2020b) para los indicadores del mercado laboral.

Chiapas, Guerrero y Oaxaca son los tres estados de México con peores condiciones de vida: menor desarrollo humano (PNUD, 2015) y mayor pobreza (Coneval, 2019), rezago social (Coneval, 2016) y marginación (Téllez et al., 2016). Al compararlos con los tres estados con menor pobreza en 2018, se observan diferencias claras en los sentidos señalados que llegan a ser muy amplias en algunos indicadores. Por ejemplo, la tasa de informalidad TIL1 es el doble en los estados pobres, donde hasta el $80 \%$ de los ocupados son laboralmente vulnerables por la naturaleza de la unidad económica para la que trabajan o tienen un vínculo laboral que no es reconocido por su fuente de trabajo. En Chiapas, Guerrero y Oaxaca menos del $18 \%$ de los ocupados cuentan con atención médica por motivo de su trabajo principal, mientras que esta característica beneficia a más del $50 \%$ de los ocupados en Coahuila, Baja California Sur y Nuevo León.

La tasa de trabajo asalariado indica el "porcentaje de la población ocupada que percibe de la unidad económica para la que trabaja un sueldo, salario o jornal, por las actividades realizadas" (Inegi, 2020a). En los estados con mayor pobreza, esta tasa se sitúa entre el 40 y el $45 \%$, mientras que en los otros alcanza el 70 u $80 \%$. El trabajo asalariado es característico de los mercados formales. De ahí surgen, nuevamente, las diferencias interregionales. Entre los no asalariados de los estados pobres hay trabajadores no remunerados, por cuenta propia, personas que dirigen su propia empresa o negocio, pero sobre todo auto-empleados a los que se puede llegar a calificar como microempresarios si el término no genera confusión respecto a su verdadera actividad: por ejemplo, comercializar en plena calle con mínima inversión en capital jugos de frutas, repostería casera, artesanías o productos textiles.

Los ingresos que obtienen de estas actividades son consecuentes con las mismas. En Chiapas, el $69 \%$ de los ocupados ingresa menos de dos salarios mínimos (290 euros al mes). En Nuevo León, el estado con menor pobreza del país, sólo el $32,6 \%$ de los trabajadores clasifica en este grupo de ingreso.

Lo contrario ocurre con el desempleo, que es menor donde predominan los mercados informales. Ante la escasez de recursos, la población tiene que trabajar para subsistir, desempeñándose en cualquier actividad que reporte algún ingreso, por exiguo que resulte. De esta manera, la TD es baja. Pero, al no poder permanecer desocupados, saturan el mercado ofreciendo productos o servicios redundantes. A consecuencia de ello, sus ingresos son escasos.

Por todo lo comentado, es muy relevante que Ios ODS de las Naciones Unidas incluyan explícitamente "el trabajo decente para todos" como parte de su objetivo 8. Este objetivo y las metas 
que lo componen se analizan a continuación a la luz de la realidad mexicana.

\section{LAS METAS DEL OBJETIVO DE TRABAJO DECENTE Y CRECIMIENTO ECONÓMICO DE LOS ODS}

El objetivo 8 de los ODS, trabajo decente y crecimiento económico, se compone de 12 metas que buscan contribuir al desarrollo económico sostenible mediante la creación de condiciones para que las personas accedan a empleos de calidad, estimulando la economía.

La primera de estas metas consiste en: "Mantener el crecimiento económico per cápita de conformidad con las circunstancias nacionales $y$, en particular, un crecimiento del producto interno bruto de al menos el $7 \%$ anual en los países menos adelantados" (Naciones Unidas, 2018: 39). Específicamente se hace referencia a la tasa de crecimiento anual del PIB real per cápita, es decir, una vez descontada la inflación. Como se ha mencionado, la creación de empleos se relaciona con el crecimiento de la producción (Okun, 1962). En México, el lento crecimiento no ha incidido tanto en el desempleo como en las condiciones laborales, que incluso han tendido a deteriorarse. Por ello, la promoción del creci- miento es una medida prioritaria (Ros, 2019b, p. 40). El problema es que México no ha mostrado capacidad para expandir su producción ni a corto ni a medio o largo plazo. En los trimestres anteriores a la emergencia sanitaria de covid-19, la economía se encontraba estancada (Mares, 2019). Según datos del Banco Mundial (2021), el PIB real per cápita de México decreció un 1,14\% en 2019, y a lo largo de toda la última década aumentó a un ritmo ligeramente superior a 1,1\% acumulativo anual, muy lejos del $7 \%$ que propone Naciones Unidas (Figura 1). De hecho, desde 1960, esa tasa sólo se ha alcanzado en dos años: 1964 (8,5\%) y 1979 (7,0\%). El último periodo de rápido crecimiento tuvo lugar de 1978 a 1981, desembocando en la crisis de la deuda (GriffithJones y Sunkel, 1986), la década perdida (Estefanía, 1984), la "inevitabilidad" del modelo neoliberal (Brieger, 2002), y la fase de lento crecimiento (Ros, 2015) que se prolonga hasta la actualidad. En el caso de México, "Mantener el crecimiento económico per cápita de conformidad con las circunstancias nacionales", implica extender una situación que ha carecido del dinamismo necesario para crear puestos de trabajo en el sector formal de la economía. Por otra parte, la meta de crecimiento a un ritmo del $7 \%$ está demasiado lejos de las posibilidades actuales del país. Ni siquiera se prevé que el "rebote" tras la crisis del covid-19 eleve la tasa más allá del 4,3\% (Morales, 2021).

Figura 1. Tasas de variación anual del PIB real per cápita y por trabajador, México 1960-2019.

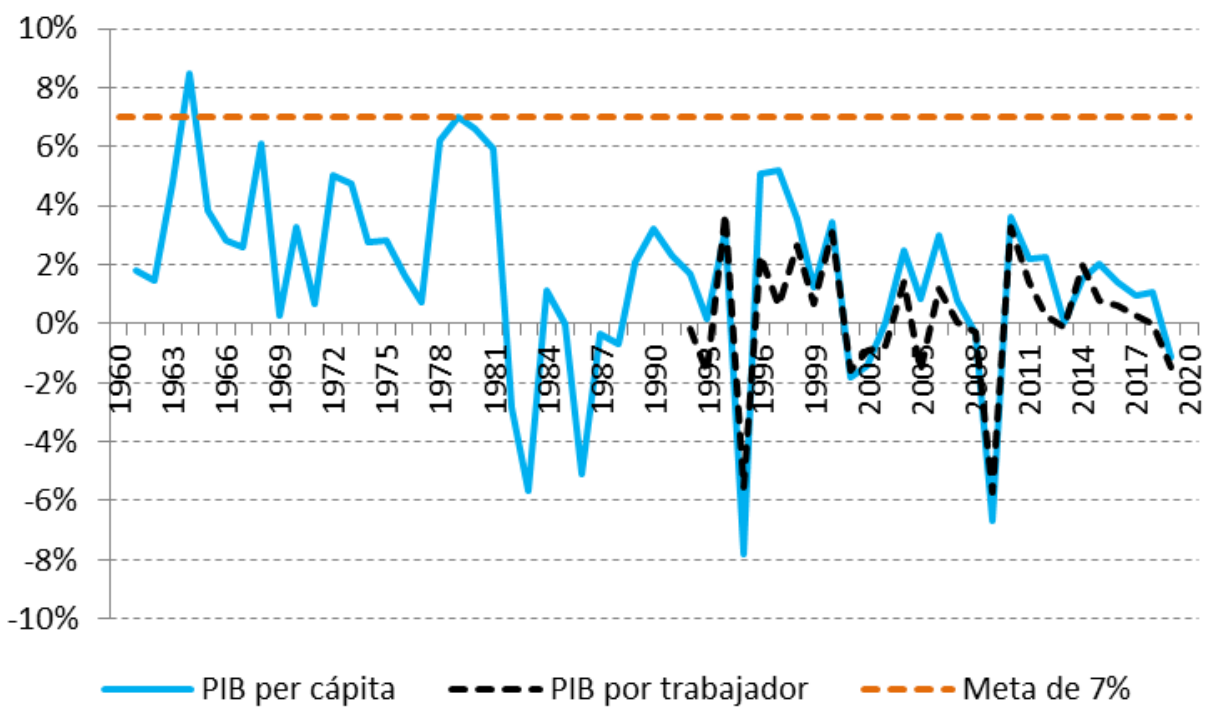

Fuente: Elaboración propia con datos del Banco Mundial (2021). 
La segunda meta busca alcanzar "niveles más elevados de productividad". Los datos del Banco Mundial (2021) sitúan a México en la posición 69 de 173 países clasificados de mayor a menor PIB por persona empleada. Con un valor de 45.764 dólares constantes, supera el promedio mundial (39.651) pero queda lejos de la media de la OCDE (95.269), de la que forma parte. En años reciéntes, se ha consolidado la idea de que es necesario incrementar la productividad para recuperar el crecimiento de la economía. Como se ha visto, el problema no es tanto la creación de empleos como la calidad de los mismos. Un gran número de trabajadores desarrollan actividades redundantes, que apenas aportan a la producción y se asocian con remuneraciones bajas, pero necesarias para continuar subsistiendo.

En la década más reciente se han propuesto cambios para mejorar la eficiencia en la asignación de recursos y elevar la productividad, por ejemplo: reformas impositivas, facilidades en el acceso al crédito, redefinición de los programas sociales para reducir los incentivos a la informalidad laboral, flexibilización del mercado de trabajo con el propósito de generar más empleo formal, promoción de la competencia en sectores oligopólicos, reforma del sistema educativo (Ros, 2013). Pero no ha habido resultados. EI PIB por trabajador, lejos de aumentar, ha permanecido estancado o incluso se ha reducido. En las últimas tres décadas, el valor máximo es el correspondiente al año 2000: 46.448 dólares (Banco
Mundial, 2021), un 1,5\% por encima del dato de 2019.

Para Levy (2019, p. 300), “La productividad de la economía cayó porque la política pública gravó al sector moderno y subsidió al de subsistencia". Para Ros (2019c, p. 281), la caída de la tasa de acumulación de capital es el factor esencial que explica el estancamiento de la economía mexicana y de su productividad. La escasez de capital físico y la baja inversión han limitado el desarrollo del sector moderno, que no ha podido generar empleos, surgiendo empresas informales de baja capitalización y productividad.

La relación entre la productividad y el producto, así como su evolución en el tiempo, se pueden analizar a partir de la siguiente descomposición factorial:

$$
\frac{Y}{P}=\frac{Y}{L} \cdot \frac{L}{P}
$$

En la que $\mathrm{Y} / \mathrm{P}$ representa el producto $(\mathrm{Y})$ por habitante $(P)$, esto es, el PIB per cápita; Y/L es el producto $(\mathrm{Y})$ por trabajador (L), es decir, la productividad del trabajo; y el cociente L/P es la proporción de trabajadores en la población total. La descomposición es cierta por construcción y, para el caso de México, arroja los resultados de la Tabla 2, calculados con datos del Banco Mundial (2021). Como se observa, el PIB per cápita aumentó durante las tres últimas décadas. No obstante, este crecimiento tuvo un ritmo lento, apenas alcanzado una tasa acumulativa anual del 0,91\% durante el periodo 1991-2019, y con estancamiento en el decenio 2000-2010.

Tabla 2. PIB per cápita, productividad y proporción de trabajadores, México 1991-2019.

\begin{tabular}{|c|c|c|c|c|}
\hline & 1991 & 2000 & 2010 & 2019 \\
\hline PIB per cápita $(\mathrm{Y} / \mathrm{P})$ & 15.367 & 17.842 & 17.875 & 19.796 \\
\hline Producto por trabajador (Y/L) & 44.039 & 46.448 & 44.105 & 45.764 \\
\hline \multirow[t]{3}{*}{ Proporción de trabajadores (L/P) } & 0,349 & 0,384 & 0,405 & 0,433 \\
\hline & \multicolumn{4}{|c|}{ Tasas de variación anual acumulativa } \\
\hline & $1991-2000$ & $2000-2010$ & $2010-2019$ & 1991-2019 \\
\hline PIB per cápita $(\mathrm{Y} / \mathrm{P})$ & $1,67 \%$ & $0,02 \%$ & $1,14 \%$ & $0,91 \%$ \\
\hline Producto por trabajador $(\mathrm{Y} / \mathrm{L})$ & $0,59 \%$ & $-0,52 \%$ & $0,41 \%$ & $0,14 \%$ \\
\hline Proporción de trabajadores (L/P) & $1,07 \%$ & $0,54 \%$ & $0,73 \%$ & $0,77 \%$ \\
\hline
\end{tabular}

Fuente: Elaboración propia con datos del Banco Mundial (2021).

Nota: EI PIB per cápita y el producto por trabajador están medidos en dólares PPA constantes de 2011.

El crecimiento del PIB per cápita puede deberse al aumento de la productividad (el mismo número de trabajadores produce más) o al hecho de que haya una mayor proporción de población produciendo. En principio, el PIB per cápita y la productividad pueden aumentar sin límite. No ocurre lo mismo con la proporción de trabaja- dores (L/P), que necesariamente debe estar comprendida entre 0 y 1 , o $100 \%$ si se expresa en porcentaje. Esta limitación no ha impedido que la expansión de la economía mexicana en las últimas décadas se haya basado más en el aumento de la proporción de trabajadores (a un 
ritmo del 0,77\% acumulativo anual) que en el crecimiento de la productividad $(0,14 \%){ }^{3}$

La propuesta de los ODS consiste en aumentar la productividad "mediante la diversificación, la modernización tecnológica y la innovación, entre otras cosas centrándose en los sectores con gran valor añadido y un uso intensivo de la mano de obra" (Naciones Unidas, 2018, p. 39). No cabe duda de que estas acciones elevarían la productividad y reactivarían el crecimiento. El problema son las condiciones estructurales prevalecientes que impiden que estos cambios ya se hayan producido. Cuando en la década de los ochenta México abandonó la estrategia de crecimiento dirigida por el mercado interno para reemplazarla por la promoción de las exportaciones, apostó por los bajos costes salariales como ventaja competitiva, y no por la productividad, situación que se ha mantenido en el tiempo hasta el punto de generar fricciones en la negociación del nuevo tratado comercial con Estados Unidos y Canadá. En concreto, fueron los líderes sindicales de estos dos países, y no los de México, los que mostraron preocupación por los bajos salarios que se pagaban en México, ya que algunas empresas trasladaban su producción al sur de la frontera reduciendo puestos de trabajo en Estados Unidos y Canadá (García, 2018).

La tercera meta se relaciona con la promoción de políticas de desarrollo "que apoyen las actividades productivas, la creación de puestos de trabajo decentes, el emprendimiento, la creatividad y la innovación, y fomentar la formalización y el crecimiento de las microempresas y las pequeñas y medianas empresas". Levy (2018, p. 298 y p. 303) argumenta que en México se ha venido haciendo lo contrario. Se ha gravado a las empresas medianas y grandes, en su mayoría formales y más productivas, que consiguen aprovechar las economías de escala y alcance, y se ha subsidiado y protegido al sector informal, de subsistencia. De esta manera, "los incentivos obstaculizan el crecimiento de las empresas productivas" y no promueven el tránsito desde la informalidad a la formalidad, sino incluso lo contrario. Atender a esta cuestión sería importante para reactivar la creación de puestos de trabajo decentes, revertir la tendencia a la baja de la productividad y recuperar el crecimiento económico.

La cuarta meta sirve para dar coherencia al conjunto de ODS al vincular este objetivo 8 con el 12 , que busca "garantizar modalidades de consumo y producción sostenibles". En concreto, promueve el consumo eficiente de recursos y desvincular el crecimiento económico de la degradación del medio ambiente "empezando por los países desarrollados" (Naciones Unidas, 2018, p. 40). El caso es que este planteamiento parece no involucrar directamente a México, que acumula varias décadas sin crecimiento, y que es miembro de la OCDE pero que, por ejemplo, en el IDH clasifica en la posición 74 de un total de 189 países (PNUD, 2020, p. 344). No obstante, los problemas medioambientales existen, como la sobreexplotación de acuíferos, el deterioro de la calidad del aire en las zonas urbanas o la rápida transformación de selvas en suelos de uso agrícola (Semarnat, 2016). Por ello, sería imprudente obviar la esencia de la propuesta.

La quinta meta se centra en la situación laboral en términos de cantidad y calidad de empleos: "lograr el empleo pleno y productivo y el trabajo decente"; poniendo especial énfasis en "la igualdad de remuneración por trabajo de igual valor". Explicita la importancia de atender a las diferencias por sexo y con respecto a los jóvenes y a las personas con discapacidad. Los datos más recientes muestran que en México la TD no es muy distinta para los hombres $(3,46 \%)$ o las mujeres (3,52\%). Las diferencias surgen con el grupo de jóvenes, donde las tasas se duplican hasta alcanzar el 6,70\% entre la población masculina de 15 a 24 años y 7,97\% entre las mujeres de la misma cohorte (Banco Mundial, 2021). Las diferencias salariales por grupos son más difíciles de evaluar porque las actividades que realizan son sustancialmente distintas, como se percibe más adelante al analizar las tasas de lesiones ocupacionales.

Los ninis ${ }^{4}$ son la población objetivo de la sexta meta, que propone "reducir considerablemente la proporción de jóvenes (de entre 15 y 24 años) que no están empleados y no cursan estudios ni reciben capacitación" (Naciones Unidas, 2018, p. 40). En el año 2011 surgió una controversia a cerca del número de ninis. Según OCDE (2011, p. 360), en México, el 24,8\% de los jóvenes de entre 15 y 29 no recibía educación, ni tenía empleo. La cifra estaba muy por encima de la de países como Dinamarca (6,6\%), Luxemburgo (7,9\%) o Noruega $(8,0 \%)$, y sólo era superada por Israel $(28,7 \%)$ y Turquía (39,6\%). La Jornada (13-09-2011) tituló "Más de siete millones de mexicanos son 'ninis'; es tercer lugar de OCDE”; El Universal (13-092011): "México, tercero de la OCDE con más ninis"; Proceso (15-09-2011): "Tiene México siete millones de 'ninis', reitera la OCDE”.

La atención se centró en la cifra agregada. Las características de estos jóvenes no fueron relevantes, aunque contenían la clave para entender el asunto. El 89\% eran mujeres, que en gran parte residían en zonas rurales. Según datos de la En- 
cuesta Nacional de la Juventud, el $72,1 \%$ de estas mujeres se dedicaba a los quehaceres del hogar. No trabajaban de cara al mercado, pero sí en el ámbito doméstico, contribuyendo con su esfuerzo a la reproducción de la economía familiar.

Esta situación evidencia algunas cuestiones que merece la pena mencionar. Por ejemplo, cabe plantearse si los conceptos que se originan en las sociedades posmaterialistas (Inglehart, 1998) son directamente aplicables o deben tener sentido semejante en sociedades con estructuras, necesidades y posibilidades claramente distintas. La idea de "ninis" es un ejemplo de ello, pero los ODS se vertebran en nociones de este tipo (Hidalgo-Capitán et al., 2019). En el caso específico de México, con un mercado de trabajo saturado, que ofrece remuneraciones bajas y condiciones precarias, no parece haber necesidad de que un mayor número de jóvenes se incorporen al mismo. A ello se suma el hecho de que "las inversiones en educación están subutilizadas" (Levy, 2018, p. 298), por lo que tampoco parece prioritario que los jóvenes sigan sobrecualificándose para empleos que no se solicitan. Se vuelve al origen del problema: el estancamiento económico y la consecuente escasez de empleos de calidad.

En cualquier caso, las medidas que se adopten para reducir la proporción de jóvenes que no están empleados y no cursan estudios ni reciben capacitación, deberían tener muy en cuenta las características reales de estos y no las habituales en otras latitudes. En 2019 empezó a operar el programa "Jóvenes construyendo el futuro", que "vincula a personas de entre 18 y 29 años de edad, que no estudian y no trabajan, con empresas, talleres, instituciones o negocios donde desarrollan o fortalecen hábitos laborales y competencias técnicas para incrementar sus posibilidades de empleabilidad a futuro" (STPS, 2021). Los jóvenes reciben capacitación durante un año, en el que disponen de seguro médico para enfermedades, maternidad y riesgos de trabajo, así como de un ingreso de 4.310 pesos mensuales (alrededor de 170 euros). Si bien aún es pronto para conocer los efectos del programa, ya ha sido noticia por irregularidades y casos de corrupción vinculados al mismo (Aveldaño et al., 2021; Hernández, 2021).

En una situación prácticamente opuesta a los ninis se encuentran los menores de edad que por diversos motivos, pero fundamentalmente por la escasez de recursos de sus familias, se ven obligados a incorporarse al mercado de trabajo. Según datos del censo de 2010 (Inegi, 2011), en
México estaban ocupados casi 248 mil jóvenes de entre doce y catorce años (el 3,8\% de ese grupo de edades), a pesar de que la fracción tercera del artículo 123 de la Constitución prohibía expresamente "la utilización del trabajo de los menores de catorce años". En 2014 este texto se reformó para incluir en la prohibición a los menores de quince años. No obstante, el número de menores ocupados en actividades laborales, lejos de reducirse, parece haberse incrementado durante la última década. El censo de 2020 (Inegi, 2021) clasifica a 800 mil jóvenes de entre doce y catorce años como ocupados, lo que supone el $12,2 \%$ de este estrato de edades. Por su parte, la Encuesta Nacional de Trabajo Infantil (ENTI) 2019 detecta hasta 2 millones de niños y 1,3 millones de niñas de 5 a 17 años que trabajan (Inegi, 2020c). Del total, 2 millones se desempeñan en actividades económicas no permitidas y los restantes 1,3 millones realizan actividades domésticas sin remuneración en sus propios hogares en condiciones no adecuadas, ya sea por soportar horarios prolongados o condiciones peligrosas. En conjunto representan el $11,5 \%$ del grupo de edades de referencia. Su distribución geográfica es bastante desigual. El estado con mayor tasa de trabajo infantil es Oaxaca, donde el $21,5 \%$ de los menores realiza alguna actividad. Le siguen Puebla y Chiapas (18,3\%). En el extremo opuesto se encuentran Baja California y Ciudad de México con tasas del 5,3\% y 5,4\%, respectivamente. Frente a estas situaciones, la séptima meta del octavo ODS busca "poner fin al trabajo infantil en todas sus formas", considerando a los niños de entre 5 y 17 años de edad. Los datos mencionados muestran que en México aún queda un largo camino por recorrer en la erradicación del trabajo infantil. Además, las tendencias recientes no invitan al optimismo. Según la ENTI, el número de jóvenes de 5 a 17 años de edad en ocupaciones no permitidas, que ponen en riesgo su salud o afectan su desarrollo físico y/o mental se redujo de 3,5 millones en 2007 a 2,3 en 2013, pero desde entonces apenas avanzó hasta los 2 millones contabilizados en 2019 (Inegi, 2020c).

La protección de los derechos laborales y la promoción de entornos de trabajo seguros son los aspectos en los que se centra la octava meta. Los indicadores asociados a esta meta son las tasas de lesiones ocupacionales mortales y no mortales, desglosadas por sexo y estatus migratorio. Esta información se puede encontrar en la base de datos de la Organización Internacional del Trabajo (OIT). Para México no hay información disponible desglosada por estatus migratorio, pero sí por sexo. La más reciente corresponde al 
año 2017 , en el que se registraron 7,5 muertes y 3.003 lesiones por cada 100.000 trabajadores. Estas tasas se han reducido desde principios de la década, cuando hubo 10 fallecimientos y 3.532 lesiones. No obstante, aún se encuentran lejos de las correspondientes a países con entornos de trabajo más seguros. Por ejemplo, en España, las lesiones laborales mortales sólo afectaron a 1,8 de cada 100.000 trabajadores y las no mortales a 3.356 (OIT, 2021).

Las diferencias por sexo son muy importantes en la tasa de fallecimientos, que se eleva hasta 11 de cada 100.000 en el caso de los hombres y apenas alcanza 1,5 entre las mujeres. No ocurre lo mismo con la tasa de lesiones laborales no mortales, mucho más parecida entre ambos sexos: 3.052 para los hombres y 2.921 para las mujeres.

El turismo sostenible es la vía que aporta la novena meta para crear puestos de trabajo y promover la cultura y productos locales. En México, entorno al 8,7\% del PIB se imputa al sector turístico (Sectur, 2020). Se trata de una cifra que en las dos décadas más recientes ha permanecido bastante estable entre el $8,2 \%$ y el $8,8 \%$, y que no cabe esperar que aumente en un futuro cercano debido a las restricciones en los viajes por la pandemia de covid-19 y a la percepción de inseguridad y violencia que en el extranjero se tiene de México.

La décima meta propone "fomentar y ampliar el acceso a los servicios bancarios, financieros y de seguros" (Naciones Unidas, 2018, p. 41). Los indicadores que se asocian a esta meta son el número de sucursales de bancos comerciales y de cajeros automáticos por cada 100.000 adultos. Ambas medidas se pueden consultar en la base de datos del Banco Mundial. En el año 2019, México disponía de 13,73 sucursales bancarias por cada 100.000 adultos. Con una ratio similar a la de Colombia $(14,48)$, Argentina $(13,45)$ o Chile $(13,05)$, se situaba en la posición 75 de un total de 147 países para los que se disponía de información. No obstante, aún se encontraba lejos de aquellos con ratios más altas. De hecho, tendría que duplicar el número de sucursales para llegar al nivel de Estados Unidos $(30,46)$ y casi cuadruplicarlo para alcanzar a España $(49,66)$. El caso es que, en México, el número de sucursales por cada 100.000 adultos dejó de incrementarse en el año 2012, cuando alcanzó el máximo de 14,89. Desde entonces, la tendencia ha sido a la reducción de esta cifra. Frente a ello, el número de cajeros automáticos ha crecido de manera rápida y sostenida durante las últimas décadas. En la actualidad, México dispone de 61,54 cajeros por cada 100.000 adultos, situándose en la posición 51 de 148 países (Banco Mundial, 2021).

Las diferencias entre regiones vuelven a ser importantes en este aspecto. La Encuesta Nacional de Inclusión Financiera (ENIF) 2018 revela que en la región noroeste el $82,3 \%$ de la población usó algún canal financiero (sucursal, cajero automático o corresponsal) en el último año (Tabla 3). Sin embargo, este porcentaje se reduce hasta el $61,6 \%$ en la región sur, donde además se observan las mayores disparidades por sexo: únicamente el $56,7 \%$ de las mujeres usó algún canal financiero frente al $67,1 \%$ de los hombres (CNBV e Inegi, 2018, p. 186).

Tabla 3. Porcentaje de población que en el último año usó algún canal financiero, 2018.

\begin{tabular}{llll}
\hline Región & Total & Mujeres & Hombres \\
\hline Noroeste & $82,3 \%$ & $81,2 \%$ & $83,6 \%$ \\
Noreste & $77,1 \%$ & $73,8 \%$ & $80,5 \%$ \\
Occidente y Bajío & $67,1 \%$ & $63,3 \%$ & $71,4 \%$ \\
Ciudad de México & $79,3 \%$ & $79,9 \%$ & $78,7 \%$ \\
Centro Sur y Oriente & $64,4 \%$ & $61,1 \%$ & $68,1 \%$ \\
Sur & $61,6 \%$ & $56,7 \%$ & $67,1 \%$ \\
\hline Nacional & $69,5 \%$ & $66,3 \%$ & $73,0 \%$ \\
\hline
\end{tabular}

Fuente: Elaboración propia con datos de CNBV e Inegi (2018, p. 186).

Nota: Los estados incluidos en cada región son: Noroeste (Baja California, Baja California Sur, Chihuahua, Durango, Sinaloa y Sonora), Noreste (Coahuila, Nuevo León, San Luis Potosí y Tamaulipas), Occidente y Bajío (Aguascalientes, Colima, Guanajuato, Jalisco, Michoacán, Nayarit, Querétaro y Zacatecas), Centro Sur y Oriente (Estado de México, Hidalgo, Morelos, Puebla, Tlaxcala y Veracruz) y Sur (Campeche, Chiapas, Guerrero, Oaxaca, Quintana Roo, Tabasco y Yucatán). 
La tendencia a la reducción del número de sucursales bancarias durante la última década y las diferencias entre regiones son dos factores a tener en cuenta ya que pueden estar limitando el acceso de la población a los servicios bancarios y financieros.

Por último, cabe mencionar dos metas adicionales que implican la coordinación de programas a nivel mundial, en concreto: "Aumentar el apoyo a la iniciativa de ayuda para el comercio" y "desarrollar y poner en marcha una estrategia mundial para el empleo de los jóvenes y aplicar el Pacto Mundial para el Empleo" (Naciones Unidas, 2018, p. 42). La Ayuda para el Comercio es un programa de apoyo de la Organización Mundial del Comercio (OMC) que presta asistencia financiera y técnica a los países en desarrollo para que puedan aprovechar mejor las oportunidades de acceso a los mercados. Por su parte, el Pacto Mundial para el Empleo es un instrumento adoptado en 2009 por la OIT que aborda las repercusiones de las crisis económicas internacionales en el ámbito social y del empleo, con el objetivo de "proveer una base acordada internacionalmente para políticas diseñadas para reducir el período entre recuperación económica y recuperación con oportunidades de trabajo decente" (OIT, 2009, p. III). En ninguna de estas estrategias México ha mostrado un especial involucramiento.

\section{REFLEXIONES FINALES}

El mercado laboral mexicano se caracteriza por bajas tasas de desempleo, altos porcentajes de informalidad laboral, bajos salarios y grandes diferencias entre las distintas regiones del país. Importantes segmentos de la población tienen la necesidad de acceder a algún ingreso, que sólo pueden generar mediante el único factor productivo del que disponen: el trabajo. Sin apenas impedimentos que restrinjan la participación activa en el mercado laboral, el autoempleo se ha constituido en la solución al mínimo dinamismo de la economía.

Desde 1981 a la fecha, la economía mexicana ha crecido a un ritmo del 0,5\% anual. Esta tasa se sitúa muy lejos del $7 \%$ que proponen los ODS. Lo grave es que en las últimas décadas la productividad se ha expandido aún menos, y las condiciones comentadas han llevado a la autogeneración de empleos redundantes en un mercado laboral informal saturado con ingresos que no permiten salir de la pobreza, sino apenas subsistir. Más de la mitad de los ocupados se encuentran en situación de informalidad. Seis de cada diez no cuentan con asistencia médica. Y la mayoría ingresa menos de 300 euros al mes. Estos porcentajes promedio se elevan sustancialmente en el sureste del país.

En este contexto, tiene sentido la propuesta de la tercera meta: "fomentar la formalización y el crecimiento de las microempresas y las pequeñas y medianas empresas" y "la creatividad y la innovación" (Naciones Unidas, 2018, p. 40); porque según Levy (2018) se ha estado haciendo lo contrario: subsidiar y proteger de manera directa e indirecta al sector informal a costa de las empresas formales.

Asimismo, se ha tratado de atraer inversiones compitiendo en costes salariales. Una estrategia que mal llevada implica entender que la pobreza de los trabajadores es una ventaja competitiva para la macroeconomía nacional. No se eligió competir en productividad, lo que hubiese dado sentido a las mejoras en formación y educación. Tampoco en infraestructura, ni en seguridad física y jurídica. El problema fue que, cuando en la década de los ochenta se decidió orientar la economía hacia el exterior, el principal y casi único socio comercial resultó ser Estados Unidos, ${ }^{5}$ con quien se puede competir en pocos asuntos.

Después de cuatro décadas de lentos avances, elevar la productividad se presenta como un factor clave para reactivar el crecimiento y la creación de empleos en el sector formal. El problema es que esto ya es conocido y se ha intentado corregir mediante reformas en el sector de la energía, en el sistema educativo, en los programas sociales, con escaso éxito. Será necesario explorar otras soluciones ya que la persistencia de esta situación hace más difícil reducir el desempleo entre los jóvenes, poner fin al trabajo infantil, que la educación sea percibida como una inversión, o reducir la tasa de informalidad y el porcentaje de trabajadores que cobran menos de un salario mínimo. Unido a ello, las amplias diferencias entre regiones invitan a actuar de manera preferente donde los rezagos son mayores con el fin de acortar las brechas existentes, mitigar los desequilibrios territoriales y procurar una mayor cohesión. 


\section{NOTAS}

1 La PEA incluye a las personas ocupadas y a las desocupadas. La TD se calcula como el porcentaje de desocupados en el total de la PEA. La tasa de empleo (o de ocupación) (TO) es el porcentaje complementario, de manera que TD+TO=100\%.

2 Por las propiedades de los logaritmos y de las tasas de variación, la suma de las tasas de variación de la productividad $(0,14 \%)$ y de la proporción de trabajadores $(0,77 \%)$ es aproximadamente igual a la tasa de variación acumulativa anual del PIB per cápita durante el mismo periodo: $0,91 \%$

3 Según datos de Naciones Unidas (2020), en el año 2019, el $77,9 \%$ de las exportaciones de México tuvo como destino Estados Unidos, mientras que el $45,3 \%$ de las importaciones de México provino de ese país.

\section{REFERENCIAS BIBLIOGRÁFICAS}

- Aveldaño, E., Charles, Á., Félix, V., Martínez, P. y Dávila, A. (2021, 3 de febrero). Roban millonada con plan de 'ninis'. Reforma.

https://www.reforma.com/aplicacioneslibre/prea cceso/articulo/default.aspx?_rval=1\&urlredirect =https://www.reforma.com/roban-millonadacon-plan-de-ninis/ar2117829?referer=-7d616165662f3a3a6262623b727a7a7279703b76 7a783a--.

- Banco Mundial (2021). Datos: indicadores. Grupo Banco Mundial.

https://datos.bancomundial.org/indicador.

- Brieger, P. (2002). De la década perdida a la década del mito neoliberal. En Gambina, J. (comp.), La globalización económico-financiera: su impacto en América Latina (pp. 341-355). Consejo Latinoamericano de Ciencias Sociales (CLACSO).

- CNBV e Inegi (2018). Encuesta Nacional de Inclusión Financiera (ENIF) 2018, principales hallazgos nacionales y regionales. Comisión Nacional Bancaria y de Valores / Instituto Nacional de Estadística y Geografía.

https://www.gob.mx/cnbv/acciones-yprogramas/medicion-de-inclusion-financiera.

- Coneval (2016). Índice de Rezago Social 2015. Presentación de resultados. Consejo Nacional de Evaluación de la Política de Desarrollo Social. https://www.coneval.org.mx/Medicion/Document s/Indice_Rezago_Social_2015/Nota_Rezago_Social_ 2015_vf.pdf.

- Coneval (2019). Resultados de pobreza en México 2018 a nivel nacional y por entidades federativas. Consejo Nacional de Evaluación de la Política de Desarrollo Social.
https://www.coneval.org.mx/Medicion/MP/Pagin as/Pobreza-2018.aspx.

- Estefanía, J. (1984, 24 de abril). América Latina, una década perdida para el crecimiento. El País. https://elpais.com/diario/1984/04/24/economia /451605605_850215.html.

- García, D. V. (2018, 4 de septiembre). El TLCAN y los bajos salarios en México. Nexos. https://economia.nexos.com.mx/?p=1846.

- Griffith-Jones, S. y Sunkel, O. (1986). Debt and development crises in Latin America: the end of an illusion. Clarendon Press.

- Hernández, S. (2021, 22 de febrero). STPS entrega becas hasta a los muertos. EI Sol de México. https://www.elsoldemexico.com.mx/mexico/politi ca/stps-entrego-becas-hasta-a-los-muertos-ninissecretaria-trabajo-jovenes-construyendo-futuro6390044.html.

- Hidalgo-Capitán, A. L., García-Álvarez, S., CubilloGuevara, A. P. y Medina-Carranco N. (2019). Los Objetivos del Buen Vivir. Una propuesta alternativa a los Objetivos de Desarrollo Sostenible. Revista Iberoamericana de Estudios de Desarrollo, 8(1), 657. https://doi.org/10.26754/ojs_ried/ijds.354.

- Inegi (2011). Censo de población y vivienda 2010. Instituto Nacional de Estadística y Geografía. https://www.inegi.org.mx/programas/ccpv/2010 /

- Inegi (2014). La informalidad laboral: Encuesta Nacional de Ocupación y Empleo: marco conceptual y metodológico. Instituto Nacional de Estadística y Geografía.

- Inegi (2020a). Encuesta Nacional de Ocupación y Empleo (ENOE), población de 15 años y más de edad: glosario. Instituto Nacional de Estadística y Geografía.

https://www.inegi.org.mx/app/glosario/default.ht $\mathrm{ml} ? \mathrm{p}=$ ENOE15.

- Inegi (2020b). Encuesta Nacional de Ocupación y Empleo (ENOE), población de 15 años y más de edad: Consulta interactiva de indicadores estratégicos (InfoLaboral), a partir de 2005. Instituto Nacional de Estadística y Geografía. https://www.inegi.org.mx/sistemas/Infoenoe/Def ault_15mas.aspx.

- Inegi (2020c). En México 3.3 millones de niños, niñas y adolescentes de 5 a 17 años trabajan: Encuesta Nacional de Trabajo Infantil (ENTI) 2019. Comunicado de Prensa, 627/20. Instituto Nacional de Estadística y Geografía.

https://www.inegi.org.mx/contenidos/saladepren sa/boletines/2020/EstSociodemo/ENTI-2019.pdf.

- Inegi (2021). Censo de población y vivienda 2020. Instituto Nacional de Estadística y Geografía. 
https://www.inegi.org.mx/programas/ccpv/2020 /.

- Inglehart, R. (1998). Modernización y posmodernización. El cambio cultural, económico y político en 43 sociedades. Centro de Investigaciones Sociológicas.

- Juárez, B. (2020, 16 de abril). Solicitudes de seguro de desempleo aumentan $40 \%$ en la CDMX. EI Economista.

https://www.eleconomista.com.mx/estados/Solici tudes-de-seguro-de-desempleo-aumentan-40-enla-CDMX-20200416-0046.html.

- Levy, S. (2018). Esfuerzos mal recompensados: la elusiva búsqueda de la prosperidad en México. Banco Interamericano de Desarrollo.

- Levy, S. (2019). Réplica a la reseña de Jaime Ros sobre 'Esfuerzos mal recompensados'. Economía UNAM, 16(46), 284-303.

http://www.scielo.org. $\mathrm{mx} /$ scielo.php?script=sci_a rttext\&pid $=$ S1665-

952X2019000100284\&lng=es\&nrm=iso.

- Mares, M. A. (2019, 31 de octubre). Estancamiento económico. El Economista.

https://www.eleconomista.com.mx/opinion/Estan camiento-economico-20191031-0020.html.

- Morales, Y. (2021, 26 de enero). FMI mejora pronóstico para PIB mexicano, apoyado por Estados Unidos. El Economista.

https://www.eleconomista.com.mx/economia/FM I-mejora-a-4.3-su-pronostico-para-el-PIB-deMexico-en-2021-20210126-0038.html.

- Naciones Unidas (2018). La agenda 2030 y los objetivos de desarrollo sostenible: una oportunidad para América Latina y el Caribe. Naciones Unidas.

- Naciones Unidas (2020). UN comtrade database Naciones Unidas. https://comtrade.un.org/data.

- Negrete, R. (2011). El indicador de la polémica recurrente: la tasa de desocupación y el mercado laboral en México. Realidad, Datos y Espacio: Revista Internacional de Estadística y Geografía, 2(1), 145-168. https://rde.inegi.org.mx/index.php/2011/01/04/e l-indicador-de-la-polemica-recurrente-la-tasa-dedesocupacion-y-el-mercado-laboral-en-mexico/.

- OCDE (2011). Education at a glance 2011: OECD indicators. OECD Publishing. http://dx.doi.org/10.1787/eag-2011-en.

- OCDE (2021). Short-term labour market statistics. OECD.Stat. Organización para la Cooperación y el Desarrollo Económicos.

https://stats.oecd.org/Index.aspx? DatasetCode $=$ S TLABOUR\#.
- Ochoa, S. M. (2005). El seguro de desempleo en México y el mundo. Reporte temático del Centro de Estudios Sociales y de Opinión Pública, 3.

- OIT (2009). Para recuperarse de la crisis: un pacto mundial para el empleo. Oficina Internacional del Trabajo.

- OIT (2021). Catálogo de datos. Organización Internacional del Trabajo. https://ilostat.ilo.org/es/data/.

- Okun, A. M. (1962). Potential GNP: its measurement and significance. Proceedings of the Business and Economic Statistics Section, 98-104.

- PNUD (2015). Índice de Desarrollo Humano para las entidades federativas, México 2015: avance continuo, diferencias persistentes. Programa de las Naciones Unidas para el Desarrollo.

- PNUD (2020). Human development report 2020: the next frontier: human development and the anthropocene. Programa de las Naciones Unidas para el Desarrollo.

- Ros, J. (2013). Algunas tesis equivocadas sobre el estancamiento económico de México. El Colegio de México / UNAM.

- Ros, J. (2015). ¿Cómo salir de la trampa del lento crecimiento y alta desigualdad? El Colegio de México / UNAM.

- Ros, J. (2019a). Los retos de la política económica. Economía UNAM, 16(46), 16-23.

http://www.scielo.org.mx/scielo.php?script=sci_a rttext\&pid=S1665-

952X2019000100016\&lng=es\&nrm=iso.

- Ros, J. (2019b). La economía mexicana en 2018: saldos, perspectivas de desaceleración, y el imperativo del crecimiento. Revista de Economía Mexicana Anuario UNAM, (4), 3-52.

http://www.economia.unam.mx/assets/pdfs/econ mex/04/01JaimeRoss.pdf.

- Ros, J. (2019c). Dinámica empresarial disfuncional y productividad estancada: una reseña del nuevo libro de Santiago Levy. Economía UNAM, 16(46), 270-283.

http://www.scielo.org.mx/scielo.php?pid=S1665952X2019000100270\&script=sci_arttext\&tlng=es.

- Sectur (2020). Cuenta satélite del turismo de México 2019, principales resultados. Secretaría de Turismo.

https://www.datatur.sectur.gob.mx/SitePages/Pro ductoDestacado3.aspx.

- Semarnat (2016). Informe de la situación del medio ambiente en México, 2015: compendio de estadísticas ambientales, indicadores clave, de desempeño ambiental y de crecimiento verde. 
Secretaría de Medio Ambiente y Recursos Naturales.

- STPS (2021). Jóvenes construyendo el futuro. Secretaría de Trabajo y Previsión Social. https://jovenesconstruyendoelfuturo.stps.gob.mx/

- Téllez, Y., Almejo, R., Hernández, A. R. y Romo, R. (2016). Índice de marginación por entidad federativa y municipio 2015. Consejo Nacional de Población. 